\title{
Corporate Yields: Effect of Credit Ratings and Sovereign Yields
}

\author{
By Julia Bevilaqua, Galina Hale, and Eric Tallman* \\ .. \\ ONLINE APPENDIX
}

Figures 1 and 2 show examples of how sovereign and corporate yields and ratings, respectively, vary over time for individual countries. Figure 3 shows how the sensitivity of corporate yields to corporate ratings changes over time for advanced and emerging economies. These lines are obtained by interacting coefficients on ratings in yield regressions with dummies for each individual year. Separate regressions are estimated for advanced and emerging economies bonds.

Table 1 presents main set of regressions without firm fixed effects. Tables 2 and 3 present more details on the interaction regressions for emerging and advanced economies, respectively. Table 2 shows regressions for sample that is split between low and high sovereign yield states.

\footnotetext{
* Bevilaqua: The Wharton School of the University of Pennsylvania, 3733 Spruce Street Philadelphia, PA 19104, jmbev@wharton.upenn.edu. Hale: Federal Reserve Bank of San Francisco, University of California at Santa Cruz, CEPR, gbhale@ucsc.edu. Tallman: Federal Reserve Bank of San Francisco, 101 Market St, San Francisco, CA 94105, ebtallman@gmail.com. The views in this paper are solely the responsibility of the authors and should not be interpreted as reflecting the views of the Board of Governors of the Federal Reserve System or any other person associated with the Federal Reserve System.
} 
Figure 1. : Corporate and sovereign yields: two examples
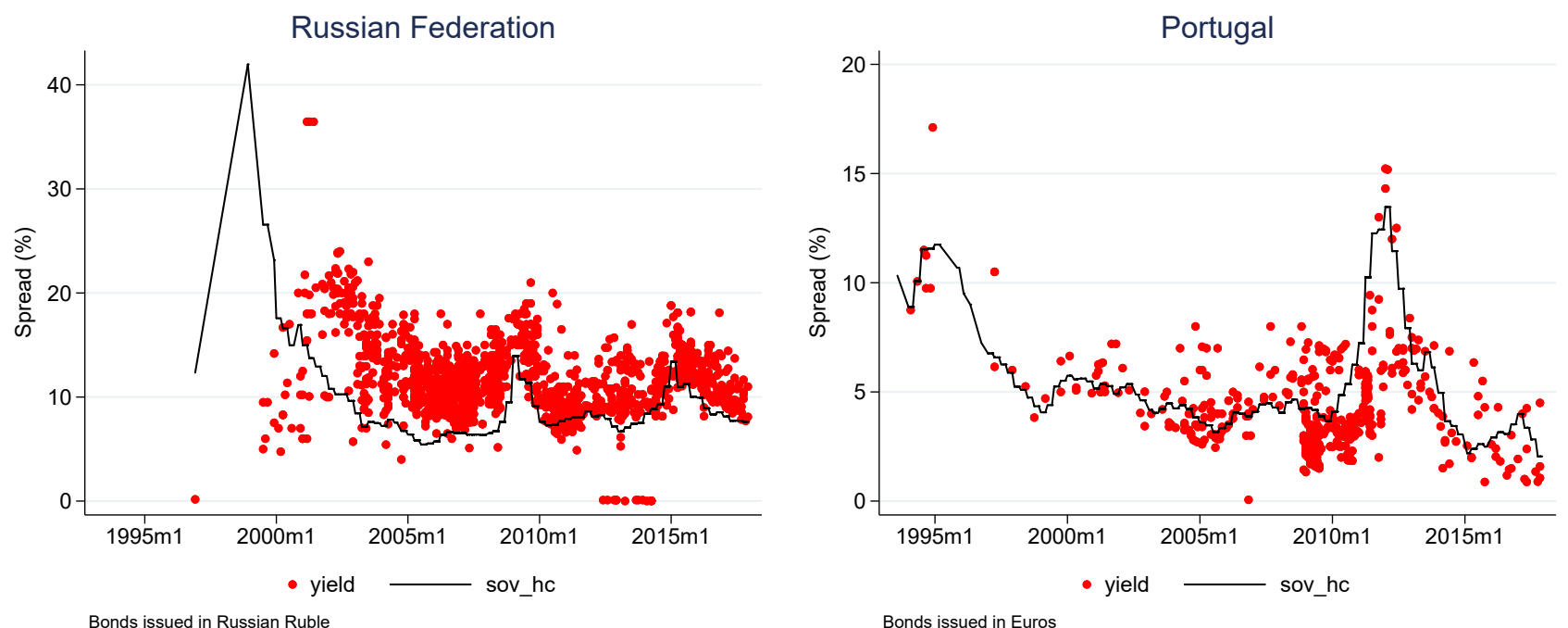

Figure 2. : Corporate and sovereign ratings: two examples
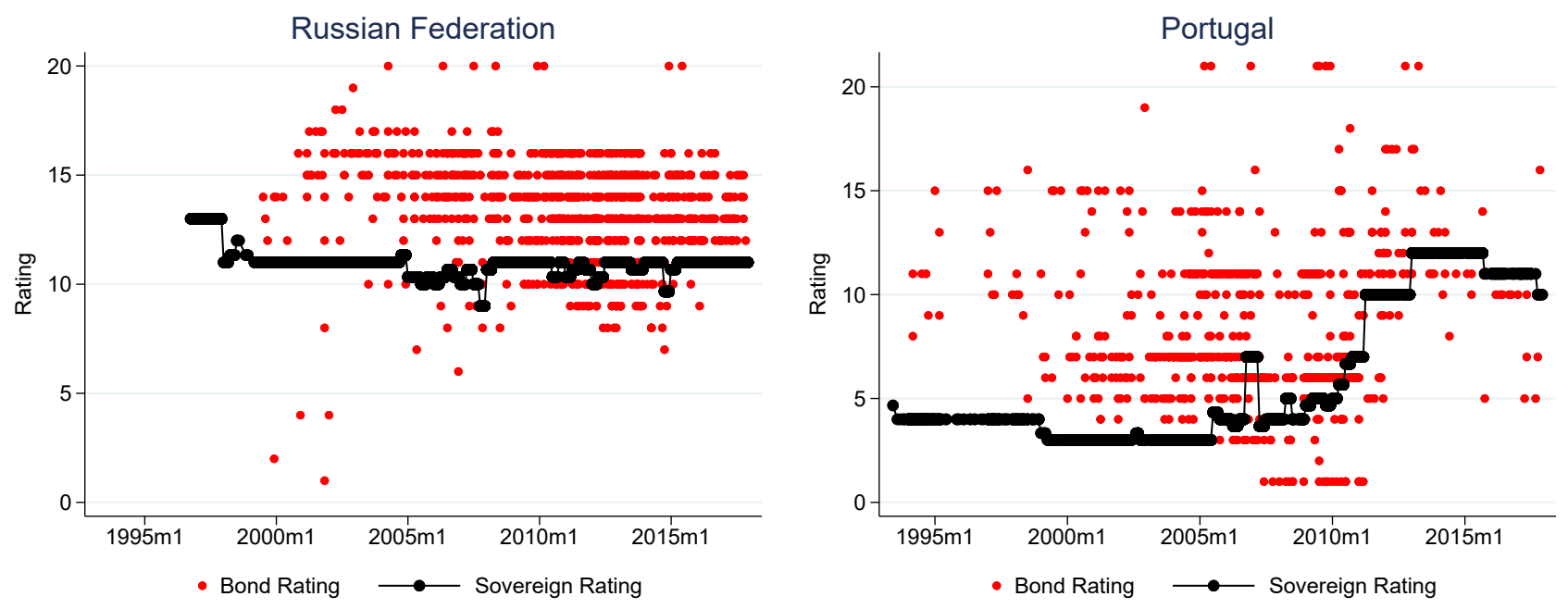

Bonds issued in Russian Ruble

Bonds issued in Euros 
Figure 3. : Sensitivity of yields to ratings over time

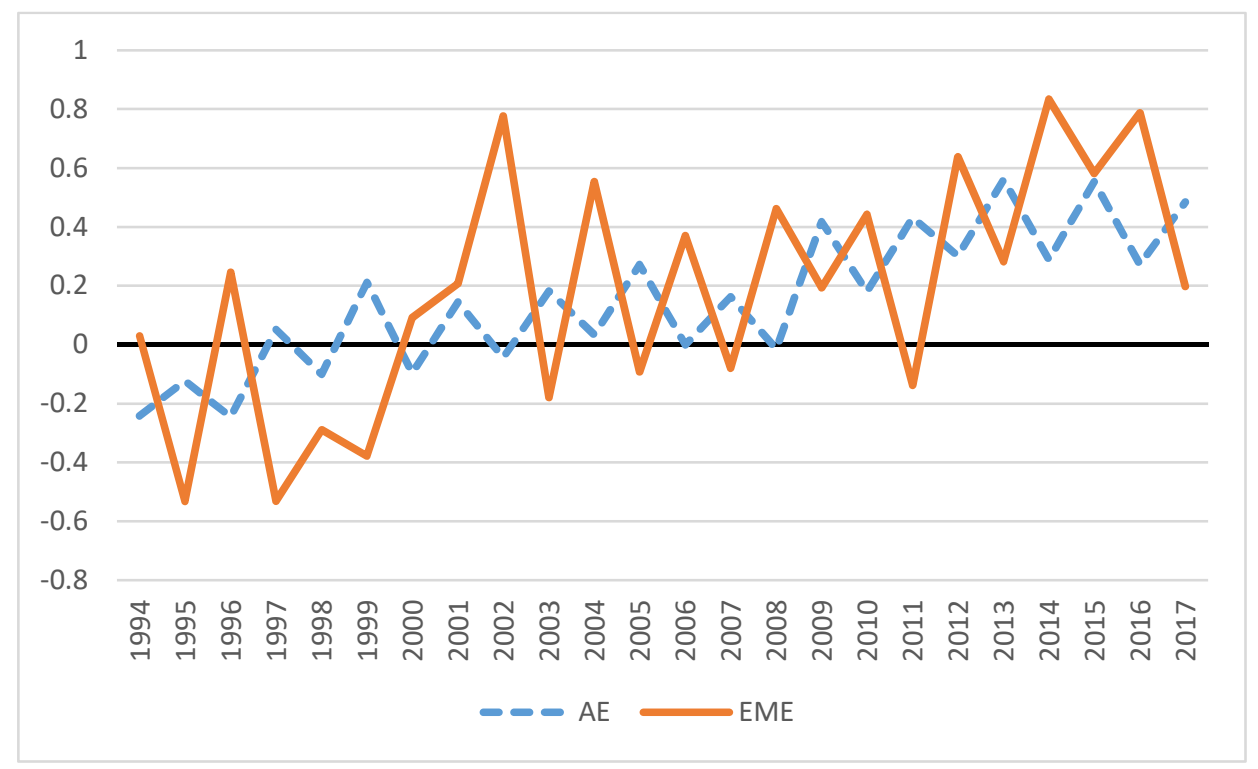

Table 1-: Yield regressions: no firm FEs

\begin{tabular}{lcccccc}
\hline & \multicolumn{3}{c}{ Emerging markets } & \multicolumn{3}{c}{ Advanced economies } \\
& $(1)$ & $(2)$ & $(3)$ & $(4)$ & $(5)$ & $(6)$ \\
\hline Bond rating & $0.68^{* * *}$ & & $0.28^{* * *}$ & $0.075^{* * *}$ & & $0.12^{* * *}$ \\
& $(0.058)$ & & $(0.026)$ & $(0.024)$ & & $(0.014)$ \\
Sovereign yield & & $1.25^{* * *}$ & $1.02^{* * *}$ & & $0.82^{* * *}$ & $0.87 * * *$ \\
& & $(0.034)$ & $(0.037)$ & & $(0.064)$ & $(0.077)$ \\
\hline Observations & 7019 & 7019 & 7019 & 35606 & 35606 & 35606 \\
Adjusted $R^{2}$ & 0.62 & 0.80 & 0.84 & 0.24 & 0.50 & 0.54 \\
Adjusted within $R^{2}$ & 0.44 & 0.71 & 0.76 & 0.021 & 0.35 & 0.41 \\
\hline
\end{tabular}

Unit of observation is individual bond. All regressions include time fixed effects.

Dependent variable is the yield of the bond.

Robust SEs clustered on country-year in all regressions.

$*(\mathrm{P}<0.10), * *(\mathrm{P}<0.05), * * *(\mathrm{P}<0.01)$. 
Table 2—: Yield regressions: emerging markets bonds

\begin{tabular}{lcccccc}
\hline & $(1)$ & $(2)$ & $(3)$ & $(4)$ & $(5)$ & $(6)$ \\
\hline Bond rating & $0.14^{* * *}$ & & $0.069^{*}$ & $0.15^{* * *}$ & & 0.017 \\
& $(0.043)$ & & $(0.037)$ & $(0.047)$ & & $(0.038)$ \\
I(below grade) & & & & -0.084 & & $-4.24^{* * *}$ \\
& & & & $(1.77)$ & & $(1.27)$ \\
Rating * I(below grade) & & & & -0.0059 & & $0.38^{* * *}$ \\
& & & & $(0.15)$ & & $(0.11)$ \\
Sovereign yield & & $0.96^{* * *}$ & $0.95^{* * *}$ & & $1.09^{* * *}$ & $1.12^{* * *}$ \\
& & $(0.037)$ & $(0.038)$ & & $(0.13)$ & $(0.14)$ \\
Sovereign yield ${ }^{2}$ & & & & & -0.0097 & -0.011 \\
& & & & & $(0.0099)$ & $(0.010)$ \\
\hline Adjusted $R^{2}$ & 0.89 & 0.92 & 0.92 & 0.89 & 0.92 & 0.92 \\
Adjusted within $R^{2}$ & 0.0099 & 0.28 & 0.28 & 0.0096 & 0.28 & 0.29 \\
\hline Unit of observation
\end{tabular}

Unit of observation is individual bond. All regressions include firm and time fixed effects.

Dependent variable is the yield of the bond. 6693 observations.

Robust SEs clustered on country-year in all regressions.

$*(\mathrm{P}<0.10), * *(\mathrm{P}<0.05), * * *(\mathrm{P}<0.01)$.

Table 3-: Yield regressions: advanced economies bonds

\begin{tabular}{lcccccc}
\hline & $(1)$ & $(2)$ & $(3)$ & $(4)$ & $(5)$ & $(6)$ \\
\hline Bond rating & $0.079 * * *$ & & $0.073^{* * *}$ & $0.063^{* * *}$ & & $0.059^{* * *}$ \\
& $(0.0090)$ & & $(0.0086)$ & $(0.0087)$ & & $(0.0086)$ \\
I(below grade) & & & & -0.50 & & -0.096 \\
& & & & $(0.48)$ & & $(0.46)$ \\
Rating * I(below grade) & & & & $0.073^{*}$ & & 0.039 \\
& & & & $(0.043)$ & & $(0.040)$ \\
Sovereign yield & & $0.42^{* * *}$ & $0.41^{* * *}$ & & $0.71^{* * *}$ & $0.70^{* * *}$ \\
& & $(0.056)$ & $(0.056)$ & & $(0.046)$ & $(0.050)$ \\
Sovereign yield & & & & & $-0.018^{* * * *}$ & $-0.018^{* * *}$ \\
& & & & & $(0.0020)$ & $(0.0023)$ \\
\hline Adjusted $R^{2}$ & 0.76 & 0.76 & 0.77 & 0.76 & 0.77 & 0.77 \\
Adjusted within $R^{2}$ & 0.018 & 0.054 & 0.069 & 0.021 & 0.066 & 0.083 \\
\hline
\end{tabular}

Unit of observation is individual bond. All regressions include firm and time fixed effects.

Dependent variable is the yield of the bond. 34252 observations.

Robust SEs clustered on country-year in all regressions.

$*(\mathrm{P}<0.10), * *(\mathrm{P}<0.05), * * *(\mathrm{P}<0.01)$. 
Table 4-: Yield regressions: by state of sovereign yields

\begin{tabular}{lcccc|cccc}
\hline \multirow{3}{*}{ Sovereign yield } & Low & Emerging markets & \multicolumn{4}{c}{ Advanced economies } \\
& $(1)$ & $(2)$ & $(3)$ & $(4)$ & $(5)$ & $(6)$ & $(7)$ & $(8)$ \\
\hline Bond rating & $0.68^{* * *}$ & 0.072 & $0.098^{* *}$ & 0.19 & $0.068^{* * *}$ & $0.18^{* * *}$ & $0.070^{* * *}$ & $0.12^{* *}$ \\
& $(0.069)$ & $(0.066)$ & $(0.048)$ & $(0.20)$ & $(0.024)$ & $(0.041)$ & $(0.0086)$ & $(0.045)$ \\
Firm fixed effects & No & No & Yes & Yes & No & No & Yes & Yes \\
\hline Observations & 6012 & 568 & 5774 & 500 & 34176 & 1099 & 32882 & 863 \\
Adjusted $R^{2}$ & 0.64 & 0.74 & 0.89 & 0.91 & 0.25 & 0.54 & 0.77 & 0.75 \\
Adjusted within $R^{2}$ & 0.45 & 0.0072 & 0.0038 & 0.0081 & 0.017 & 0.12 & 0.014 & 0.045 \\
\hline
\end{tabular}

Unit of observation is individual bond. All regressions include time fixed effects.

Dependent variable is the yield of the bond.

Robust SEs clustered on country-year in all regressions.

$*(\mathrm{P}<0.10), * *(\mathrm{P}<0.05), * * *(\mathrm{P}<0.01)$. 